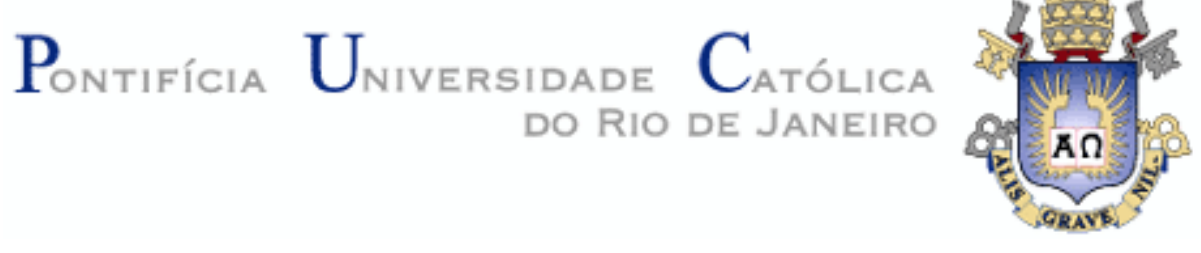

Jacileide de Almeida Serafim

\title{
CONCEPÇÃO E IMPLANTAÇÃO DE GRUPOS AUTOGERENCIÁVEIS: ANÁLISE DE DOIS CASOS INDUSTRIAIS
}

\section{Dissertação de Mestrado}

Dissertação apresentada como requisito parcial para obtenção do título de Mestre pelo Programa de PósGraduação em Engenharia de Produção da PUCRio.

Orientadora: Márcia Cristina Esteves Agostinho

Rio de Janeiro Fevereiro de 2005 


\title{
CONCEPÇÃO E IMPLANTAÇÃO DE GRUPOS AUTOGERENÇIÁVEIS: ANÁLISE DE DOIS CASOS INDUSTRIAIS
}

\begin{abstract}
Dissertação apresentada como requisito parcial para obtenção do título de Mestre pelo Programa de PósGraduação em Engenharia de Produção da PUC-Rio. Aprovada pela Comissão Examinadora abaixo assinada.
\end{abstract}

Márcia Cristina Esteves Agostinho Orientadora Departamento de Engenharia Industrial - PUC - Rio

\section{Antônio Glauter Teófilo Rocha}

Luiz Felipe Roris Rodriguez Scavarda do Carmo Departamento de Engenharia Industrial - PUC - Rio

Maria Ângela Campelo de Melo Departamento de Engenharia Industrial - PUC - Rio

José Eugênio Leal Coordenador(a) Setorial de Pós-graduação e Pesquisa do Centro Técnico Científico - PUC-Rio 
Todos os direitos reservados. É proibida a reprodução total ou parcial do trabalho sem autorização da universidade, da autora e do orientador.

\section{Jacileide de Almeida Serafim}

Graduou-se em Engenharia Química pela Universidade Federal do Pará. Desenvolveu trabalhos de iniciação científica em Engenharia de Alimentos, onde apresentou trabalhos em congressos e outros eventos científicos da área. Depois de graduada ingressou em um projeto de pesquisa e desenvolvimento na área de Engenharia de Alimentos e trabalhou em uma Organização não Governamental com desenvolvimento local sustentável.

Ficha Catalográfica

Serafim, Jacileide de Almeida

Concepção e implantação de grupos autogerenciáveis : análise de dois casos industriais / Jacileide de Almeida Serafim ; orientadora: Márcia Cristina Esteves Agostinho. - Rio de Janeiro : PUC, Departamento de Engenharia Industrial, 2005.

$102 \mathrm{f.} ; 30 \mathrm{~cm}$

Dissertação (mestrado) - Pontifícia Universidade Católica do Rio de Janeiro, Departamento de Engenharia Industrial.

Inclui referências bibliográficas.

1. Engenharia industrial - Teses. 2. Autonomia. 3. Grupos autogerenciáveis. 4. Organização do trabalho. I. Agostinho, Márcia Cristina Esteves. II. Pontifícia Universidade Católica do Rio de Janeiro. Departamento de Engenharia Industrial. III. Título. 


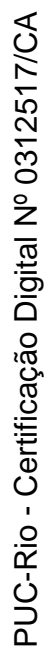

Para Raquel, minha mãe. 


\section{Agradecimentos}

- A Deus por tamanha bondade e cuidado para comigo dando-me força e capacidade para realizar meus objetivos;

- A minha orientadora Márcia Agostinho, pela valiosa orientação acadêmica, amizade, incentivo e por tudo que me ensinou;

- A minha família que, mesmo de longe, me deu amor e apoio em todos os momentos na realização desse sonho;

- Ao meu namorado, Igor, pelo apoio, paciência, carinho e amizade em todos os momentos, bons e maus;

- Aos professores do curso de pós-graduação do Departamento de Engenharia Industrial, em especial à professora Maria Ângela Campelo de Melo que sempre que precisei me acolheu e ajudou;

- Às empresas Ambev e Michelin por permitirem a realização do estudo em suas fábricas;

- Ao CNPq pelo apoio financeiro;

- Aos meus amigos Alexandre e Juliana que sempre me apoiaram;

- Aos funcionários do DEI e colegas da PUC que direta ou indiretamente colaboraram na realização desse trabalho. 


\section{RESUMO}

Serafim Jacileide de Almeida. Concepção e Implantação de Grupos Autogerenciáveis: Análise de dois Casos Industriais. Rio de Janeiro, 2005. 102p. Dissertação de Mestrado - Departamento de Engenharia Industrial, Pontifícia Universidade Católica do Rio de Janeiro.

A presente dissertação tem como objetivo identificar e compreender o processo de concepção e implantação de grupos autogerenciáveis na produção, por meio da análise de dois casos de implantação desses grupos, nas empresas Ambev e Michelin. Busca-se primeiramente analisar as abordagens que introduziram o conceito de grupos autogerenciáveis, ressaltando suas características. Os casos são descritos de forma a mostrar a situação das empresas antes das mudanças, bem como as etapas mais relevantes nos processos de concepção e implantação do novo modelo. Uma análise comparativa dos dois casos foi realizada com vistas a compreender os fatores importantes na condução dos processos de concepção e implantação de grupos autogerenciáveis. Conclui-se, a partir da análise, que a definição dos modelos foi influenciada pela observação de casos concretos de implantação em outras empresas, não levando em consideração aspectos teóricos. Os processos de implantação apresentam muitas semelhanças. A distinção, por sua vez, apresenta-se em algumas especificidades das etapas. Por fim, foram destacados alguns fatores que podem ser indutivos ou limitantes para o desenvolvimento dos grupos autogerenciáveis.

\section{Palavras-Chave:}

Autonomia; grupos autogerenciáveis; organização do trabalho. 


\section{ABSTRACT}

Serafim Jacileide de Almeida. Conception and Implementation of Self-managing Groups: Analysis of two Industrial Cases. Rio de Janeiro, 2005. 107p. MSc. Dissertation - Departamento de Engenharia Industrial, Pontifícia Universidade Católica do Rio de Janeiro.

The present dissertation aims at identifying and understanding the process of conception and implementation of self-managing groups in production. Following the methodology of case study, the experiences of two companies are observed: AmBev and Michelin. The main intent is to understand each approach, describing the organizational context before the changes towards self-managing groups and also the key steps for implementing the new model. Then, the two cases are comparatively interpreted, leading to some conclusions about what should be the most important factors to be taken into account when implementing self-managing groups. It is worth noting that, in both companies, the conception of the respective models emerges from observations of a variety of concrete cases related to the experiences of other plants or companies. They were hardly influenced by theory. Although the two approaches share many similar steps, some significant differences can be highlighted. Finally, such similarities and differences are analyzed in order to grasp factors that can induce or constrain the development of self-managing groups.

\section{Key-words}

Autonomy; self-managing groups; organization of the work. 


\section{Sumário}

1.INTRODUÇÃO 13

2.ORGANIZAÇÃO DO TRABALHO EM GRUPOS AUTOGERENCIÁVEIS NA PRODUÇÃO 16

2.1. Abordagem sociotécnica 16

2.1.1. Origem 16

2.1.2. Principais conceitos desenvolvidos 17

2.1.3. Níveis de intervenção 23

2.2. Modelo Japonês de gestão $\quad 24$

2.3. Considerações acerca da concepção e implantação de equipes autogerenciáveis $\quad 27$

2.4. Considerações finais 32

3. METODOLOGIA DE PESQUISA 33

3.1. Escolha metodológica 33

3.2. Escolha das empresas $\quad 34$

3.3. Procedimento metodológico 34

3.3.1. Condução das visitas e entrevistas 35

3.4. Limitações da pesquisa 36

4. O CASO AMBEV 37

4.1. A empresa $\quad 37$

4.2. Processo produtivo 38

4.3. Modelo organizacional 40

4.3. I Organização fabril: projeto manufatura 40

4.3.2. Políticas de RH: encarreiramento, avaliações, reconhecimento e indicadores de desempenho 43

4.4. Processo de mudança para o trabalho em grupos 46

4.5. Concepção e implantação de grupos autogerenciáveis 46

4.6. Dinâmica de funcionamento do grupo 51 
4.8. Considerações finais 58

5. O CASO MICHELIN 59

5.1. A empresa 59

5.2. Processo produtivo 61

5.3. Modelo organizacional 63

5.3.1. Organização fabril 63

5.3.2. Políticas de RH: encarreiramento, avaliações, reconhecimento e indicadores de desempenho 64

5.4. Processo de mudança para o trabalho e grupos 64

5.5. Concepção e implantação de grupos autogerenciáveis 66

5.6. Dinâmica de funcionamento dos grupos 72

5.7. Aspirações dos operadores com o novo modelo 76

5.8. Considerações finais 78

6. ANÁLISE DOS CASOS 80

6.1. Grupos semi-autônomos ou grupos no estilo japonês? 80

6.2. Motivação para a implantação 83

6.3. Estruturas organizacionais 85

6.4. Processo de mudança 86

6.5. Aspectos do projeto de concepção 87

6.6. Implantação 88

7. CONCLUSÕES 91

8. REFERÊNCIAS BIBLIOGRÁFICAS 96

$\begin{array}{ll}\text { ANEXO I } & 99\end{array}$ 


\section{Lista de figuras}

Figura 1. Processo Produtivo de Cerveja $\quad 39$

Figura 2. Processo Produtivo de Refrigerantes 40

Figura 3. Formação dos grupos a partir das células 51

Figura 4 Etapas do Processo Produtivo de Pneu 62

Figura 5. Estrutura Organizacional antes e após a redução hierárquica 66

Figura 6. Formação dos Grupos 72 


\section{Lista de Quadros}

Quadro 1. Princípios de projeto do trabalho 20

Quadro 2. Propriedades do trabalho 22

Quadro 3. Estratégias de implantação dos grupos 32

Quadro 4. Critérios referentes à política de encarreiramento 45

Quadro 5. Atividades de gestão atribuídas do grupo 53

Quadro 6. Autonomia dos grupos $\quad 55$

$\begin{array}{ll}\text { Quadro 7. Atividades de gestão do grupo } & 73\end{array}$

Quadro 8. Autonomia dos grupos 76 


\section{Lista de SIGLAS}

ANTEAG - Associação Nacional dos Trabalhadores em Autogestão e Participação Acionária

CCQs - Círculos de Controle de Qualidade

CIPA - Comissão Interna de Prevenção de Acidentes

GMR - Grupo de Melhoria da Rotina

GPs - Grupos de Progresso

ISO - International Organization for Standardization

OR - Organizations Responsabilisantes

PDCA - Plan, Do, Check, Action

PEF - Programa de Excelência Fabril

SPF - Serviço de Formação para a América Latina

TQM - Total Quality Managent

TPM - Total Productive Maintenance

UGB - Unidade Gerencial Básica

RF - Responsável de Formação 